\title{
REGULAÇÕES PSICOSSOCIAIS NA ORGANIZAÇÃO \\ DE CRENÇAS SOBRE A INTELIGÊNCIA: RELAÇÕES \\ ENTRE REPRESENTAÇÕES SOCIAIS DE INTELIGÊNCIA \\ E CONCEPÇÕES PESSOAIS DE INTELIGÊNCIA ${ }^{1}$
}

\author{
Virgílio Amaral ${ }^{2}$
}

\begin{abstract}
Resumo: No presente trabalho analisam-se os processos que regulam a organização de crenças sobre a inteligência em adolescentes, nomeadamente a influência da "familiaridade" com o objecto de representação sobre tais crenças. Os resultados mostram que os sujeitos para os quais as diferenças interindividuais de inteligência constituem algo de inexplicável tendem a explicá-la de modo biológico e a naturalizá-la, sob a forma de uma teoria implícita dos dons (desigualmente) naturais.

Investigam-se, ainda, as relações entre o princípio da "familiaridade" com a inteligência, as representações sociais da inteligência e as concepções pessoais de inteligência. Os resultados mostram que as crenças que postulam a existência de "inteligências alternativas" às definidas pela instituição escolar predizem negativamente as concepções estáticas e positivamente as concepções dinâmicas. Verifica-se, também, que a crença nas "desigualdades naturais" da inteligência é hegemónica, sendo partilhada quer pelos sujeitos estáticos, quer pelos dinâmicos.
\end{abstract}

Palavras-chave: inteligência, representações sociais, concepções pessoais.

Psychosocial regulation in the organization of intelligence beliefs: relations between social representations of intelligence and personal conceptions of intelligence (Abstract): In the present work we analyse the processes that regulate the organization of beliefs about intelligence in adolescents, namely the influence of the "familiarity" with the object of the representation. Results show that subjects for whom intelligence is an unfamiliar topic explain it in a biological way and represent it as a natural gift.

We also research the relations among the "familiarity" principle, the social representations of intelligence, and the personal conceptions of intelligence. Results

\footnotetext{
${ }^{1}$ A correspondência relativa a este artigo deve ser enviada para Virgílio Amaral, Instituto Superior de Psicologia Aplicada, Rua Jardim do Tabaco, 34, 1100-049, Lisboa. E-mail: vamaral@ispa.pt.

2 Instituto Superior de Psicologia Aplicada. Unidade de Investigação em Psicologia Cognitiva, do Desenvolvimento e da Educação.
} 
show that some beliefs about "alternative intelligences" to the school definitions predict negatively static conceptions and positively dynamic conceptions. We also find that a belief in the "natural inequality" of intelligence is a hegemonic lay theory, shared both by static and dynamic subjects.

Keywords: intelligence, social representations, personal conceptions.

\section{Introdução}

Alguns estudos realizados no âmbito da teoria das concepções pessoais de inteligência evidenciam que estas se podem formar em função da influência social exercida por certas concepções científicas ou explícitas acerca da natureza da inteligência, que a concebem e explicam, ou como sendo inata e inscrita nos genes (portanto, não mutável), ou como atributo dinâmico cujo incremento seria possível através de factores ambientais propícios ou do esforço dos indivíduos (para uma revisão, ver Dweck, 1999).

O estudo das relações entre, por um lado, as representações sociais da inteligência - crenças culturais, sejam de proveniência científica ou não -, nomeadamente como fontes de influência em termos de teorias implícitas socialmente disponíveis, e, por outro lado, as concepções pessoais de inteligência, isto é, o modo como as pessoas entendem a sua própria inteligência, parece-nos constituir um objectivo pertinente para uma compreensão do modo como variáveis de nível macrossocial - particularmente, as representações sociais - podem afectar variáveis de nível micro ou individual exactamente, as concepções pessoais de inteligência. Trata-se de um aspecto referido, por exemplo, por Faria (1998), a propósito das possíveis diferenças culturais entre contextos nacionais - no caso, Portugal e Estados Unidos da América - que podem explicar as diferenças entre concepções pessoais de inteligência, no que se refere à variável género, existentes entre os dois países.

No que respeita à teoria das representações sociais, situamos a nossa abordagem na perspectiva sociodinâmica, teórica e empiricamente formalizada por autores ligados à Universidade de Bolonha (Carugati \& Selleri, 1998; Emiliani \& Molinari, 1995; Mugny \& Carugati, 1985), que, a par das propostas de Doise e colaboradores (Clémence, Doise \& Lorenzi-Cioldi, 1994; Doise, 1989), teorizam as representações sociais como "princípios organizadores" de crenças, concepções e teorias implícitas, cujo teor e respectiva articulação ancora nas diversas representações ou posições simbólicas e em determinadas dinâmicas sociocognitivas, como seja o grau de familiaridade com o objecto de representação. Em particular, as investigações de Mugny e Carugati (1985) e de Carugati e Selleri (1998) sobre a inteligência mostram que a ausência de familiaridade dos sujeitos com 
aquele tópico leva à organização de crenças naturalizantes e essencialistas, segundo as quais a inteligência constitui um dom natural, e à valorização de dimensões biológicas.

\section{Objectivos e Hipóteses}

Os objectivos desta pesquisa são, por um lado, o de analisar, em adolescentes, a relação preditiva das dimensões representacionais da inteligência relativamente às concepções pessoais acerca da inteligência e, por outro lado, o de investigar o impacto da "não familiaridade" com o objecto representacional sobre as dimensões da representação da inteligência, bem como sobre as concepções pessoais de inteligência.

Com base na literatura já referida, formulamos neste estudo as seguintes hipóteses:

1. Como primeira hipótese, consideramos que a "não familiaridade" com a inteligência conduza à sua "naturalização" sob formas biológicas e inatistas, como decorre do estudo de Mugny e Carugati (1985), embora este último com adultos;

2. A nossa segunda hipótese é a de que as dimensões representacionais biológicas e inatistas - o mesmo é dizer teorias implícitas "rígidas" ou menos maleáveis - acerca da inteligência predigam positivamente uma concepção estática e negativamente uma concepção dinâmica, pelo "fixismo" e "finalismo" que implicam;

3. De igual modo, mas como terceira hipótese, consideramos que a "não familiaridade" com o objecto prediga positivamente uma concepção estática (fixista e finalista) e negativamente uma concepção dinâmica (mais maleável) da inteligência.

\section{Método}

\section{Sujeitos}

A uma amostra de 144 adolescentes, com idades compreendidas entre os 15 e os 19 anos, de ambos os sexos, foram administrados um questionário estruturado sobre as representações sociais da inteligência e um questionário estruturado sobre as concepções pessoais de inteligência.

As variáveis sexo, sucesso académico e meio sociocultural de origem constituem variáveis de caracterização da amostra.

No Quadro 1, apresentamos uma síntese da caracterização da amostra, de acordo com a sua segmentação em função daquelas variáveis. 
Quadro 1 - Caracterização da amostra

\begin{tabular}{llccc}
\hline Meio sociocultural & \multicolumn{1}{c}{ Sexo } & $\begin{array}{c}\text { Com } \\
\text { Reprovação }\end{array}$ & $\begin{array}{c}\text { Sem } \\
\text { Reprovação }\end{array}$ & Totais \\
\hline \multirow{3}{*}{ Baixo } & Masculino & 4 & 1 & $5(3,4 \%)$ \\
& Feminino & 2 & 6 & $8(5,5 \%)$ \\
& Total & 6 & 7 & $13(9,0 \%)$ \\
\hline \multirow{2}{*}{ Médio-baixo } & Masculino & 4 & 12 & $16(11,1 \%)$ \\
& Feminino & 4 & 8 & $12(8,3 \%)$ \\
& Total & 8 & 20 & $28(19,4 \%)$ \\
Médio-alto & Masculino & 4 & 10 & $14(9,7 \%)$ \\
& Feminino & 2 & 19 & $21(14,5 \%)$ \\
& Total & 6 & 29 & $35(24,3 \%)$ \\
\hline \multirow{2}{*}{ Alto } & Masculino & 6 & 28 & $34(23,6 \%)$ \\
& Feminino & 5 & 29 & $34(23,6 \%)$ \\
Totais & Total & 11 & 57 & $68(47,2 \%)$ \\
\hline
\end{tabular}

\section{Material e Procedimento}

O questionário sobre as representações sociais da inteligência que utilizámos funda-se, maioritariamente, num questionário utilizado e aferido numa investigação precedente (Amaral, 2005). Incluímos, também, alguns indicadores que se mostraram relevantes num estudo exploratório sobre os conteúdos representacionais da inteligência em crianças e adolescentes (Amaral, Vala \& Carugati, 2002), a saber:

- Inteligência e interesses dos jovens e dos adolescentes (item na negativa) - "Ir ao cinema ou ouvir música não tem nada a ver com a inteligência porque perde-se tempo de estudo";

- Saber Procedimental - Corresponde a scripts de rotinas diárias: "Ser inteligente é saber fazer coisas (como pôr uma cassete num vídeo, ligar a luz, etc.)";

- Representação tautológica da inteligência - "As pessoas inteligentes decoram melhor e mais coisas porque são inteligentes";

-Inteligência e Masculinidade - "Pode-se não ser muito inteligente para os estudos mas ser para o desporto";

-Inteligência e Feminilidade - "Pode-se não ser muito inteligente para os estudos mas ser para as artes";

-Inteligência Social - "Ser inteligente é ter comportamentos correctos"; 
- Representação biológica da inteligência - "As pessoas inteligentes decoram melhor e mais coisas porque têm mais espaço no cérebro".

Por sua vez, de forma a operacionalizar o grau de familiaridade com o tópico da representação ("inteligência") e a crença de que a inteligência constitui um "dom natural", utilizámos os seguintes itens:

- "A inteligência é algo de misterioso e inexplicável” (do questionário utilizado por Mugny \& Carugati, 1985);

- "Alguns já nascem muito inteligentes, outros pouco inteligentes" indicador que pretende operacionalizar a "teoria" dos "dons naturais".

Quanto ao questionário sobre as Concepções Pessoais de Inteligência, foi administrado o instrumento, construído, adaptado e validado para o contexto português por Faria (1995).

Por fim, refira-se que, para evitar o "efeito de ordem", os dois instrumentos foram administrados de forma alternada e as administrações foram efectuadas com uma semana de intervalo.

\section{Apresentação dos resultados}

Os itens do questionário de representações sociais da inteligência, com excepção dos indicadores de dimensões representacionais específicas, como os que foram já mencionados, foram alvo de factorização com o método da máxima verosimilhança e rotação varimax.

Utilizando o mesmo método e tipo de rotação, procedeu-se também à análise factorial dos itens do questionário sobre Concepções Pessoais de Inteligência.

De seguida, apresentamos as estruturas factoriais obtidas, bem como a sua interpretação.

\section{Estrutura Factorial das Concepções Pessoais de Inteligência}

No Quadro 2, apresentamos a estrutura factorial das concepções pessoais de inteligência.

Com base nos itens com saturação superior em cada factor (igual ou superior a 0,50), foram construídos indicadores formados pela média das variáveis com saturações iguais ou superiores a 0,50 . Por razões de conveniência discursiva, passaremos a designar tais indicadores também como factores. 
Quadro 2 - Estrutura factorial das Concepções Pessoais de Inteligência

\begin{tabular}{|c|c|c|}
\hline Itens & $\begin{array}{c}\text { F1: } \\
\text { "Estático" }\end{array}$ & $\begin{array}{c}\text { F2: } \\
\text { "Dinâmico" }\end{array}$ \\
\hline $\begin{array}{l}\text { Se não és tão inteligente quanto desejas, não podes fazer muito } \\
\text { para mudar isso. }\end{array}$ & 0,66 & $-0,09$ \\
\hline Não podes mudar muito a inteligência que possuis. & 0,66 & $-0,12$ \\
\hline Quando te esforças, mostras que és pouco inteligente. & 0,61 & $-0,13$ \\
\hline $\begin{array}{l}\text { A procura de novas soluções ou estratégias para resolver uma } \\
\text { tarefa demonstra que não consegues dominá-la. }\end{array}$ & 0,61 & $-0,15$ \\
\hline $\begin{array}{l}\text { A má realização numa tarefa que tens que realizar pode fazer } \\
\text { pensar que és pouco inteligente. }\end{array}$ & 0,53 & $-0,28$ \\
\hline $\begin{array}{l}\text { Por mais que te esforces nunca conseguirás mudar a tua } \\
\text { inteligência de base. }\end{array}$ & 0,53 & $-0,41$ \\
\hline $\begin{array}{l}\text { Tu tens uma certa quantidade de inteligência e não podes fazer } \\
\text { muito para a mudar. }\end{array}$ & 0,53 & $-0,47$ \\
\hline $\begin{array}{l}\text { Podes aprender coisas novas, mas não podes realmente mudar a } \\
\text { tua inteligência de base. }\end{array}$ & 0,51 & 0,06 \\
\hline Não podes aumentar a inteligência com que nasceste. & 0,48 & 0,11 \\
\hline $\begin{array}{l}\text { Os erros que cometes devem ser esquecidos, porque mostram } \\
\text { que és pouco inteligente. }\end{array}$ & 0,48 & $-0,29$ \\
\hline $\begin{array}{l}\text { Realizar bem uma tarefa pode permitir desenvolver a tua } \\
\text { inteligência. }\end{array}$ & $-0,43$ & 0,42 \\
\hline $\begin{array}{l}\text { As dificuldades e desafios que encontras impedem-te de } \\
\text { desenvolver a tua inteligência. }\end{array}$ & 0,43 & $-0,40$ \\
\hline $\begin{array}{l}\text { Os resultados obtidos nas tarefas que realizas são mais } \\
\text { importantes do que aquilo que aprendes com estas tarefas. }\end{array}$ & 0,35 & 0,13 \\
\hline $\begin{array}{l}\text { Aquilo que aprendes com as tarefas é mais importante do que os } \\
\text { resultados nelas obtidos. }\end{array}$ & 0,35 & $-0,16$ \\
\hline $\begin{array}{l}\text { A boa preparação para uma tarefa pode ser uma maneira de } \\
\text { provares aos outros que és inteligente. }\end{array}$ & $-0,17$ & 0,79 \\
\hline $\begin{array}{l}\text { Realizar bem uma tarefa pode permitir desenvolver a tua } \\
\text { inteligência. }\end{array}$ & $-0,24$ & 0,69 \\
\hline $\begin{array}{l}\text { Os erros que cometes podem ser uma oportunidade para } \\
\text { desenvolver a tua inteligência. }\end{array}$ & $-0,15$ & 0,66 \\
\hline $\begin{array}{l}\text { Os desafios e as dificuldades que enfrentas ajudam-te a } \\
\text { desenvolver a tua inteligência. }\end{array}$ & $-0,08$ & 0,57 \\
\hline $\begin{array}{l}\text { Conseguires bons resultados nas tarefas que realizas é uma prova } \\
\text { da tua inteligência. }\end{array}$ & 0,30 & $-0,47$ \\
\hline O esforço permite-te tornar mais inteligente. & 0,20 & 0,47 \\
\hline Quando aprendes coisas novas a tua inteligência aumenta. & $-0,06$ & 0,38 \\
\hline Podes tornar-te mais inteligente se quiseres. & $-0,06$ & 0,37 \\
\hline Com esforço podes conseguir mudar a tua inteligência de base. & $-0,15$ & 0,34 \\
\hline Podes fazer alguma coisa para mudar a tua inteligência. & 0,02 & 0,28 \\
\hline $\begin{array}{l}\text { Realizar bem uma tarefa permite mostrar aos outros que és } \\
\text { inteligente. }\end{array}$ & $-0,08$ & $-0,18$ \\
\hline
\end{tabular}


Por sua vez, no Quadro 3 constam os valores próprios de cada factor, as percentagens de variância total $(39,14 \%)$ e de cada factor, bem como a fidelidade de cada factor testada pelo alpha de Cronbach.

Quadro 3 - Valores próprios dos factores, variância explicada e valores de alpha

\begin{tabular}{lcc}
\hline & \multicolumn{2}{c}{ Factores } \\
\cline { 2 - 3 } & $\begin{array}{c}\text { F 1: } \\
\text { "Estático" }\end{array}$ & $\begin{array}{c}\text { F2: } \\
\text { "Dinâmico" }\end{array}$ \\
\hline Valores de alpha & 0,60 & 0,77 \\
Valores próprios & 6,22 & $2,38 \%$ \\
\% de variância explicada & $28,30 \%$ & $10,83 \%$ \\
\hline \% de variância total & \multicolumn{3}{c}{$39,14 \%$} \\
\hline
\end{tabular}

O primeiro factor, "Estático", reúne itens que revelam uma concepção fixista da inteligência, acentuando que esta não é susceptível de mudança, nem em função da dimensão esforço, nem por meio da mestria de possíveis estratégias para resolver tarefas.

O segundo factor mostra uma percepção maleável e incremental da inteligência, incluindo indicadores que mostram que a inteligência pode ser desenvolvida por meio do feedback das aprendizagens, correspondendo ao factor "Dinâmico".

\section{Estrutura Factorial das Representações Sociais da Inteligência}

No Quadro 4, apresentamos a estrutura factorial das representações sociais da inteligência.

Tal como anteriormente, com base nos itens com saturações superiores em cada factor (igual ou superior a 0,50 ), construíram-se indicadores constituídos pela média de tais itens ou variáveis. Por razões de conveniência discursiva, designaremos tais indicadores também como factores.

Por sua vez, no Quadro 5 constam os valores próprios de cada factor original/real, as percentagens de variância total $(57,96 \%)$ e também as explicadas por cada factor, bem como a fidelidade de cada factor testada pelo alpha de Cronbach. 
Quadro 4 - Estrutura factorial das Representações Sociais da Inteligência

\begin{tabular}{|c|c|c|c|c|c|c|}
\hline Itens & $\begin{array}{l}\text { F1: } \\
\text { Cogni- } \\
\text { tivo }\end{array}$ & $\begin{array}{l}\text { F2: Estu- } \\
\text { do/ Moti- } \\
\text { vação }\end{array}$ & $\begin{array}{l}\text { F3: Inteli- } \\
\text { gências } \\
\text { Alter- } \\
\text { nativas }\end{array}$ & $\begin{array}{l}\text { F4: } \\
\text { Saber }\end{array}$ & $\begin{array}{l}\text { F5: } \\
\text { Altruís- } \\
\text { mo }\end{array}$ & $\begin{array}{l}\text { F6: } \\
\text { Inatismo }\end{array}$ \\
\hline $\begin{array}{l}\text { Os inteligentes são melhores a matemática porque } \\
\text { compreendem mais depressa. }\end{array}$ & 0,77 & 0,17 & 0,03 & 0,00 & 0,05 & $-0,07$ \\
\hline $\begin{array}{l}\text { Os inteligentes são melhores a matemática, porque } \\
\text { fazem melhor e mais rapidamente as contas. }\end{array}$ & 0,75 & 0,03 & 0,05 & 0,09 & 0,04 & 0,05 \\
\hline $\begin{array}{l}\text { As pessoas inteligentes decoram melhor e mais coisas, } \\
\text { porque têm mais capacidade de memória. }\end{array}$ & 0,61 & 0,20 & 0,04 & 0,12 & 0,06 & $-0,07$ \\
\hline $\begin{array}{l}\text { As pessoas inteligentes compreendem mais depressa, } \\
\text { porque têm mais capacidades. }\end{array}$ & 0,54 & 0,05 & $-0,11$ & 0,18 & $-0,10$ & 0,19 \\
\hline $\begin{array}{l}\text { Alguns já nascem muito inteligentes, outros pouco } \\
\text { inteligentes. }\end{array}$ & 0,36 & 0,17 & 0,02 & $-0,12$ & $-0,07$ & 0,19 \\
\hline O que os inteligentes aprendem são coisas da escola. & 0,15 & 0,71 & $-0,05$ & $-0,05$ & 0,05 & 0,08 \\
\hline $\begin{array}{l}\text { Os inteligentes têm melhores notas, porque estudam } \\
\text { mais. }\end{array}$ & 0,06 & 0,67 & 0,04 & 0,23 & $-0,12$ & 0,07 \\
\hline $\begin{array}{l}\text { Os inteligentes são melhores nas disciplinas em que é } \\
\text { preciso estudar muito, porque estudam muito ou mais } \\
\text { que os outros. }\end{array}$ & 0,16 & 0,55 & 0,11 & 0,19 & 0,02 & $-0,07$ \\
\hline $\begin{array}{l}\text { As pessoas inteligentes aprendem nas escolas ou } \\
\text { universidades. }\end{array}$ & 0,05 & 0,43 & 0,03 & 0,12 & 0,05 & 0,00 \\
\hline $\begin{array}{l}\text { As pessoas que têm mais dificuldades em aprender são } \\
\text { menos inteligentes, porque não estudam. }\end{array}$ & 0,09 & 0,42 & 0,03 & 0,13 & 0,05 & 0,00 \\
\hline $\begin{array}{l}\text { Pode-se não ser inteligente para os estudos, mas ser } \\
\text { para as artes. }\end{array}$ & 0,13 & 0,02 & 0,91 & 0,04 & 0,02 & 0,08 \\
\hline $\begin{array}{l}\text { Pode-se não ser inteligente para os estudos, mas ser } \\
\text { para o desporto. }\end{array}$ & 0,05 & 0,16 & 0,65 & $-0,04$ & 0,07 & 0,11 \\
\hline $\begin{array}{l}\text { As pessoas inteligentes não tiram melhores notas, } \\
\text { porque isso tem a ver com esforço, estudo e trabalho. }\end{array}$ & $-0,14$ & $-0,11$ & 0,37 & 0,00 & 0,18 & 0,24 \\
\hline $\begin{array}{l}\text { Decorar melhor ou mais coisas não tem nada a ver com } \\
\text { a inteligência, porque o importante é compreender, não } \\
\text { é decorar. }\end{array}$ & $-0,10$ & $-0,26$ & 0,27 & 0,00 & $-0,04$ & 0,06 \\
\hline Quanto mais saber se tem, mais inteligente se é. & 0,11 & 0,18 & $-0,07$ & 0,78 & $-0,09$ & 0,03 \\
\hline Quanto mais cultura se tem, mais inteligente se é. & 0,04 & 0,10 & 0,05 & 0,45 & 0,03 & $-0,03$ \\
\hline Ser inteligente é saber muito. & 0,08 & 0,28 & 0,04 & 0,33 & 0,07 & $-0,01$ \\
\hline Ajudar os outros é uma acção inteligente. & $-0,02$ & 0,03 & 0,13 & $-0,03$ & 0,98 & 0,11 \\
\hline $\begin{array}{l}\text { É inteligente ajudar os outros, porque podemos precisar } \\
\text { da ajuda deles. }\end{array}$ & 0,05 & 0,13 & 0,07 & 0,31 & 0,34 & 0,21 \\
\hline $\begin{array}{l}\text { Já se nasce inteligente, porque já temos algumas } \\
\text { capacidades à nascença. }\end{array}$ & 0,06 & 0,06 & 0,11 & $-0,11$ & 0,07 & 0,75 \\
\hline $\begin{array}{l}\text { Já nascemos com alguma inteligência que se } \\
\text { desenvolve se a trabalharmos. }\end{array}$ & 0,03 & $-0,14$ & 0,19 & 0,12 & 0,10 & 0,53 \\
\hline
\end{tabular}


Quadro 5 - Valores próprios dos factores, variância explicada e valores de alpha

\begin{tabular}{lcccccc}
\hline \multicolumn{7}{c}{ Factores } \\
\hline & $\begin{array}{c}\text { F 1: } \\
\text { Cogni- } \\
\text { tivo }\end{array}$ & $\begin{array}{c}\text { F 2: } \\
\text { Estudo/ } \\
\text { Motivação }\end{array}$ & $\begin{array}{c}\text { F 3: } \\
\text { Inteligências } \\
\text { Alternativas }\end{array}$ & $\begin{array}{c}\text { F 4: } \\
\text { Saber }\end{array}$ & $\begin{array}{c}\text { F 5: } \\
\text { Altruís- } \\
\text { mo }\end{array}$ & $\begin{array}{c}\text { F 6: } \\
\text { Ina- } \\
\text { tismo }\end{array}$ \\
\hline $\begin{array}{l}\text { Valores } \\
\text { de alpha }\end{array}$ & 0,77 & 0,71 & 0,75 & - & - & 0,56 \\
$\begin{array}{l}\text { Valores } \\
\text { próprios } \\
\text { \% de } \\
\text { variância } \\
\text { explicada }\end{array}$ & 3,75 & 2,53 & 1,94 & 1,43 & 1,30 & 1,18 \\
$\begin{array}{l}\text { \% variância } \\
\text { total }\end{array}$ & $17,89 \%$ & $12,08 \%$ & $9,27 \%$ & $6,85 \%$ & $6,20 \%$ & $5,66 \%$ \\
\hline
\end{tabular}

O primeiro factor foi denominado "Cognitivo", já que para o mesmo concorrem competências cognitivas, mostrando também uma forte valorização da inteligência lógico-matemática.

O segundo factor é o factor clássico de "Estudo/Motivação", agregado à instituição escolar, que já obtivéramos em pesquisa anterior.

O terceiro factor foi designado "Inteligências Alternativas", pois liga a inteligência a dimensões alternativas, como o desporto ou as artes, e não ao estudo.

O quarto factor revela, tal como o próprio e único item que o satura, o "Saber".

O quinto factor mostra uma dimensão de "Altruísmo".

Finalmente, o sexto e último factor, já encontrado em investigação precedente (Amaral, 2005), corresponde ao "Inatismo". Devemos aqui notar que o item que operacionaliza a teoria dos "dons naturais" não satura o factor "Inatismo". De facto, este reenvia para uma noção mais piagetiana maturação de capacidades, desenvolvimento da inteligência pelo exercício da mesma - do que para o acaso genético. É possível que esta representação derive da influência duma pedagogia piagetiana, amplamente difundida pelos agentes e instituições educativos, sobre o modo como os adolescentes pensam a natureza da inteligência.

Efeitos da "não familiaridade" sobre as dimensões representacionais

Para testar a nossa primeira hipótese, procedemos à recodificação, em dois níveis, do item que operacionaliza a "não familiaridade" com a inteligência, obtendo-se, assim, a percepção subjectiva dos sujeitos, de maior ou menor familiaridade, com o objecto representacional. Seguidamente, proce- 
demos a um teste $t$-Student, considerando como variável independente o indicador da "não familiaridade" com a inteligência e como variáveis dependentes o indicador da dimensão representacional biológica da inteligência ("As pessoas inteligentes decoram melhor e mais coisas porque têm mais espaço no cérebro") e o que operacionaliza a teoria dos dons naturais (“Alguns já nascem muito inteligentes, outros pouco inteligentes").

Os resultados (Quadro 6) mostram médias significativamente diferentes nos indicadores da dimensão biológica da inteligência $(t=-3,85$, $p=0,00, g l=92)$ e da teoria dos "dons naturais" $(t=-2,00, p=0,048$, $g l=92$ ), em função da variável independente "não familiaridade".

Quadro 6 - Médias e desvios-padrão em função da maior ou menor familiaridade com a Inteligência

\begin{tabular}{lccc}
\hline "Familiaridade" & $\boldsymbol{N}$ & Média & Desvio-padrão \\
\hline $\begin{array}{l}\text { "Representação biológica da inteligência" } \\
\text { "maior familiaridade" }\end{array}$ & 41 & 1,46 & 0,59 \\
"menor familiaridade" & 53 & 2,22 & 1,15 \\
\hline "Teoria dos dons naturais" & & & \\
"maior familiaridade" & 41 & 2,63 & 1,19 \\
"menor familiaridade" & 53 & 3,07 & 0,93 \\
\hline
\end{tabular}

Como podemos verificar pelas médias, os sujeitos que percepcionam menor familiaridade com a inteligência valorizam mais a dimensão representacional biológica da inteligência, tal como hipotetizámos. Por sua vez, são também os sujeitos que percepcionam menor familiaridade com a inteligência que tendem a "naturalizá-la" mais em termos de "dons naturais", o que corrobora os resultados de Mugny e Carugati (1985).

\section{Relações entre Representações Sociais e Concepções Pessoais de Inteligência}

De modo a estudar as relações entre as representações sociais da inteligência e as concepções pessoais de inteligência, procedemos a análises de regressão múltipla, tendo como variáveis independentes a "não familiaridade" com a inteligência, os factores extraídos da análise factorial e os itens/indicadores complementares das já anteriormente referidas dimensões representacionais. 
Como variáveis dependentes, consideraram-se as concepções pessoais "Estática" e "Dinâmica" da inteligência.

A título exploratório, consideramos ainda na equação de regressão, e como variável independente, o item que operacionaliza a teoria dos "dons naturais".

Regressão múltipla para o factor "Estático"

Quadro 7 - Variáveis no modelo, valores beta, valores t e significância

\begin{tabular}{lccc}
\hline & $\boldsymbol{\beta}$ & $\boldsymbol{t}$ & $\boldsymbol{p}$ \\
\hline "Familiaridade" & 0,08 & 0,95 & 0,34 \\
"Interesses dos adolescentes e jovens" & 0,09 & 0,98 & 0,32 \\
"Inteligência Social" & 0,13 & 1,44 & 0,15 \\
"Tautologia" & $-0,14$ & $-1,36$ & 0,17 \\
"Saber Procedimental" & 0,03 & 0,37 & 0,70 \\
"Representação biológica da Inteligência" & 0,03 & 0,39 & 0,69 \\
"Teoria dos dons naturais" & 0,27 & 2,96 & 0,00 \\
Factor 1 - "Cognitivo" & 0,11 & 1,05 & 0,29 \\
Factor 2 - "Estudo/Motivação" & 0,10 & 1,12 & 0,26 \\
Factor 3 - "Inteligências Alternativas" & $-0,17$ & $-2,07$ & 0,04 \\
Factor 4 - "Saber" & $-0,11$ & $-1,30$ & 0,19 \\
Factor 5 - "Altruísmo" & $-0,12$ & $-1,41$ & 0,15 \\
Factor 6 - "Inatismo" & $-0,06$ & $-0,78$ & 0,43 \\
\hline $\boldsymbol{R}^{\mathbf{2}=\mathbf{0 , 1 3}}$ & & &
\end{tabular}

Como podemos verificar pelo Quadro 7, o item que operacionaliza a "não familiaridade" com a inteligência não prediz significativamente a escala estática, ao contrário do que hipotetizámos. Mais ainda, também ao contrário das nossas hipóteses, a representação biológica da inteligência não prediz a escala estática. Já a "teoria dos dons naturais" prediz positivamente a escala estática, mas, como veremos adiante, prediz também positivamente a escala dinâmica. Finalmente, o Factor 3 - relativo às "Inteligências Alternativas" - prediz negativamente as concepções pessoais estáticas.

Desta forma, as nossas hipóteses relativamente à escala estática são, globalmente, infirmadas pelos resultados obtidos. Contudo, a discussão dos resultados obtidos só poderá ser realizada a seguir, após a apresentação dos resultados da regressão múltipla relativa à escala dinâmica (Quadro 8).

\section{Regressão múltipla para o Factor "Dinâmico"}

Tal como afirmámos atrás, a "teoria dos dons naturais" prediz positivamente o factor dinâmico, tal como acontecia para o factor estático, o que 
significa que é uma teoria implícita largamente aceite pelos adolescentes da nossa amostra, independentemente do que possam pensar acerca da sua própria inteligência.

Quadro 8 - Variáveis no modelo, valores beta, valores t e significância

\begin{tabular}{lrrc}
\hline & $\boldsymbol{\beta}$ & $\boldsymbol{t}$ & $\boldsymbol{p}$ \\
\hline "Familiaridade" & $-0,06$ & $-0,83$ & 0,40 \\
"Interesses dos adolescentes e jovens" & $-0,06$ & $-0,73$ & 0,46 \\
"Inteligência Social" & 0,09 & 1,68 & 0,24 \\
"Tautologia" & 0,01 & 0,19 & 0,84 \\
"Saber Procedimental" & 0,29 & 3,64 & 0,00 \\
"Representação biológica da Inteligência" & $-0,30$ & $-3,32$ & 0,00 \\
"Teoria dos dons naturais" & 0,17 & 2,02 & 0,04 \\
Factor 1 - "Cognitivo" & 0,03 & 0,38 & 0,70 \\
Factor 2 - "Estudo/Motivação" & 0,08 & 1,05 & 0,26 \\
Factor 3 - "Inteligências Alternativas" & 0,10 & 1,28 & 0,20 \\
Factor 4 - "Saber" & 0,17 & 2,13 & 0,03 \\
Factor 5 - "Altruísmo" & 0,28 & 3,36 & 0,00 \\
Factor 6 - "Inatismo" & 0,12 & 1,54 & 0,12 \\
\hline $\boldsymbol{R}^{\mathbf{2}=\mathbf{0 , 2 6}}$ & \multicolumn{3}{c}{} \\
\hline
\end{tabular}

Ao contrário do que hipotetizámos, a "não familiaridade com a inteligência" não tem qualquer impacto sobre o factor dinâmico.

Verificamos, ainda, que as componentes representacionais que apelidámos de "Saber Geral/Cultura" (Factor 4) e "Altruísmo" (Factor 5) predizem positivamente as concepções pessoais dinâmicas. $\mathrm{O}$ mesmo sucede no que concerne ao indicador "Saber Procedimental". Ou seja, parece que, para os sujeitos que se percepcionam como intelectualmente mais "ágeis", dimensões representacionais que não sejam determinadas pelos critérios escolares constituem dimensões relevantes e determinantes das suas percepções pessoais.

Já o indicador da "Representação biológica da Inteligência" prediz negativamente as concepções pessoais dinâmicas, confirmando, parcialmente, a nossa segunda hipótese.

\section{Discussão dos resultados}

Os resultados do teste à hipótese de que a (não) familiaridade com o objecto da representação - a Inteligência - constitui um princípio organizador de crenças biológicas e inatistas sobre a inteligência confirmam os 
resultados de Mugny e Carugati (1985). Os nossos resultados, bem como os da pesquisa mencionada de Mugny e Carugati (1985), revelam, sobretudo, que os sujeitos tendem a adoptar uma crença "natural" e essencialista - de que já se nasce ou não inteligente, e é nessa medida que se é, por essência e "naturalmente", inteligente ou não - quando manifestam ausência de "familiaridade" com o objecto de representação.

Esta constatação leva-nos a considerar interessante que, em pesquisas futuras, acerca de outros objectos sociais de representação, se procure analisar se a "não familiaridade" com aqueles objectos pode levar, de modo sistemático ou tendencial, ao recurso a "teorias implícitas naturais" que conduzam a uma categorização essencialista dos sujeitos sociais.

Um ponto a reter, dos dados que apresentámos, é o de que a crença "natural", socialmente partilhada, nas desigualdades inatas de inteligência parece surgir como uma dimensão representacional hegemónica, quer em relação a sujeitos que se percepcionam como "dinâmicos", quer em relação aos que se percepcionam como "estáticos", em termos da inteligência individual. Assim, são requeridos novos e sistemáticos estudos para que se possa chegar a conclusões mais sólidas sobre a predominância, na cultura portuguesa, de uma representação tão "natural" como arbitrária.

Relativamente ao facto de as nossas hipóteses não serem, na sua totalidade, confirmadas, pensamos que dois factores podem explicar o sucedido.

Por um lado, há que considerar que o impacto das representações sociais sobre as percepções individuais da inteligência pode ser mediado por processos psicossociais específicos entre um nível mais macro e cultural (as representações sociais da inteligência) e um nível mais micro ou pessoal (as concepções pessoais de inteligência). Este dado sugere a pertinência da realização de novos trabalhos empíricos sobre variáveis psicossociais mediadoras do impacto de crenças sociais de nível macro sobre crenças individuais de nível micro.

Por outro lado, temos também que atender ao facto de a escala que afere as concepções pessoais de inteligência, em particular a escala "estática", poder incidir sobre as competências escolares, e as variáveis representacionais em jogo (variáveis independentes) se poderem encontrar relacionadas com outras dimensões de inteligência que não a escolar. Na realidade, também os resultados de uma investigação de Selleri, Carugati e Bison (1994) mostraram que, no âmbito escolar, as representações de um colega inteligente e de um colega bom aluno eram afectadas pelas dimensões requeridas pela instituição escolar - precisamente uma inteligência "escolar".

No entanto, verificámos que dimensões representacionais alternativas aos critérios institucionais/escolares determinam positivamente concepções pessoais dinâmicas, o que sugere que os sujeitos "dinâmicos" podem recorrer a alguns factores extra-escolares quando procuram entender uma inteli- 
gência mais maleável e incremental, o que parece poder contribuir para definir as suas percepções pessoais no plano intelectual.

\section{Referências}

Amaral, V. (2005). "Inteligências": estudo das representações sociais da inteligência - Perspectivas "desenvolvimentistas" e de género e dinâmicas representacionais. Tese de Doutoramento. Lisboa: Instituto Superior de Ciências do Trabalho e da Empresa.

Amaral, V., Vala, J., \& Carugati, F. (2002). Perspectivas “desenvolvimentistas” e de género acerca das representações sociais da inteligência. In J. Vala, M. Garrido e P. Alcobia (Orgs.), Percursos da investigação em psicologia social e organizacional (Vol. I). Lisboa: Colibri.

Carugati, F., \& Selleri, P. (1998). Social representations and development: expert's and parent's discourses about a puzzling issue. In U. Flick (Ed.), The psychology of the social. Cambridge: Cambridge University Press.

Clémence, A., Doise, W., \& Lorenzi-Cioldi, F. (1994). Prises de positions et príncipes organisateurs de représentations sociales. In Ch. Guillemi (Ed.), Structures et transformations des représentations sociales. Paris: Delachaux et Niestlé.

Doise, W. (1989). Attitudes et représentations sociales. In D. Jodelet (Ed.), Les représentations sociales. Paris: P.U.F.

Dweck, C. S. (1999). Self-theories: Their role in motivation, personality and development. Philadelphia: Psychology Press.

Emiliani, F., \& Molinari, L. (1995). Rappresentazioni e affetti: Carattere e interazione nello sviluppo dei bambini. Milano: Raffaello Cortina.

Faria, L. (1995). Desenvolvimento diferencial das concepções pessoais de inteligência durante a adolescência. Tese de Doutoramento. Porto: Faculdade de Psicologia e de Ciências da Educação da Universidade do Porto.

Faria, L. (1998). Diferenças de género nos padrões de realização e nas concepções pessoais de inteligência: especificidades do contexto cultural português. Psicologia: Teoria, Investigação e Prática, 3, 73-82.

Mugny, G., \& Carugati, F. (1985). L'intelligence au pluriel. Cousset: Delval.

Selleri, P., Carugati, F., \& Bison I. (1994). Compagni intelligenti e compagni bravi a scuola. Rassegna di Psicologia, XI (2), 29-52. 